\title{
Resonance electron attachment to natural polyphenolic compounds and their biological activity
}

\author{
${ }^{1}$ S.A. Pshenichnyuk ${ }^{1,2, \dagger}$, N.L. Asfandiarov ${ }^{1,3}$, A.S. Vorob’ev ${ }^{1}$, E.P. Nafikova ${ }^{1}$, A.S. Komolov ${ }^{2}$, \\ Y.N. Elkin ${ }^{4}$, N.I. Kulesh ${ }^{4}$, A. Modelli ${ }^{5}$ \\ †sapsh@anrb.ru
}
${ }^{1}$ Institute of Molecule and Crystal Physics, Ufa Research Centre, Russian Academy of Sciences, Prospekt Oktyabrya 151, 450075 Ufa, Russia
${ }^{2}$ Physics Faculty, St. Petersburg State University, Uljanovskaja 1, 198504 St. Petersburg, Russia
${ }^{3}$ Bashkir State Pedagogical University, Oktyabrskoy Revolutsii st. 3a, 450000 Ufa, Russia
${ }^{4}$ Pacific Institute of Bioorganic Chemistry, Far Eastern Branch of Russian Academy of Sciences, Prospekt 100 Let
Vladivostoku, 159, 690022 Vladivostok, Russia
${ }^{5}$ Università di Bologna, Dipartimento di Chimica "G. Ciamician", via Selmi 2, 40126 Bologna, Italy

Resonance low-energy (0-14 eV) electron attachment to natural polyphenolic stilbenes possessing antioxidant properties, namely resveratrol and piceatannol, was investigated by means of dissociative electron attachment spectroscopy. Experimental findings were assigned on base of density functional theory (DFT) calculations of energies and symmetry of vacant molecular orbitals. It was found that characteristic decay of the molecular negative ions of compounds under investigation under gasphase conditions can be associated with elimination of neutral $\mathrm{H}_{2}$ molecule and formation of quinone-like structure bearing excess electron. These fragment species can be responsible for ability of polyphenolic compounds to scavenge free radicals in the living cells. The gas-phase data were extrapolated to reactions in cellular environment by means of DFT calculations using polarizable continuum model approach. A molecular mechanism for antioxidant activity of polyphenolic compounds in proximity to the mitochondrial respiratory chain under conditions of excess negative charge was suggested. Namely, it is thought that molecular hydrogen, known for its selective antioxidant properties, can be efficiently generated via attachment of electrons ("leaked" from the respiratory chain into mitochondrial intermembrane space) to polyphenolic compound and may be responsible for its antioxidant activity. The corresponding negative fragment, i.e., quinone bearing an excess negative charge, can serve as electron carrier and can return the captured electron back to the respiration cycle. The number of hydroxyl substituents and their relative positions on aromatic rings of polyphenolic molecule are crucial for the present molecular mechanism, because these properties determine dissociative electron attachment cross-section.

Keywords: polyphenols, antioxidant activity, electron transfer, $\mathrm{H}$ atom abstraction, dissociative electron attachment, molecular mechanism.

\section{Резонансный захват электронов молекулами природных полифенольных соединений и их биологическая активность}

\author{
${ }^{1}$ Пшеничнюк С.А. ${ }^{1,2, \dagger}$, Асфандиаров Н.Л. ${ }^{1,3}$, Воробьев А.С. ${ }^{1}$, Нафикова Е.П. ${ }^{1}$, \\ Комолов А.С. ${ }^{2}$, Елькин Ю.Н. ${ }^{4}$, Кулеш Н.И. ${ }^{4}$, Моделли А. ${ }^{5}$ \\ †sapsh@anrb.ru

\begin{abstract}
${ }^{1}$ Институт физики молекул и кристаллов УНЦ РАН, Пр. Октября, 151, 450075 Уфа, Россия
${ }^{2}$ Санкт-Петербургский государственный университет (физический факультет), ул. Ульяновская, 1, 198504 Санкт-Петербург, Россия
\end{abstract}

\footnotetext{
1 In memoriam of my father, Pshenichnyuk Anatoly Ivanovich (1949-2015), whose personal example and ideas revealed to me the importance and beauty of research work, the purport and enduring value of disclosing of the mysteries of Nature. Посвящается памяти моего отца, Пшеничнюка Анатолия Ивановича (1949-2015), своим примером и мыслями открывшему мне значение и красоту исследовательской работы, смысл и непреходящую ценность проникновения в тайны Природы.
} 
${ }^{3}$ Башкирский государственный педагогический университет им. М. Акмуллы, ул. Октябрьской революции, 3а, 450000, Уфа, Россия.

${ }^{4}$ Тихоокеанский институт биоорганической химии им. Г.Б. Елякова ДВО РАН, Пр. 100 лет Владивостоку, 159, 690022, Владивосток, Россия

${ }_{5}^{5}$ Болонский Университет (химический факультет), ул. Селми, 2, 40126, Болонья, Италия

Методом спектроскопии диссоциативного захвата электронов исследован резонансный захват медленных (0-14 эВ) электронов молекулами полифенольных стильбенов природного происхождения - ресвератрола и пицеатаннола, проявляющих антиоксидантные свойства. Экспериментальные результаты интерпретированы с помощью расчётов энергий и симметрии вакантных молекулярных орбиталей методами теории функционала плотности (ТФП). Обнаружено, что характерным распадом отрицательных молекулярных ионов исследованных соединений в газовой фазе является выброс нейтральной молекулы $\mathrm{H}_{2}$ и образование хиноидной структуры, несущей избыточный электрон. Указанные фрагменты могут быть ответственны за способность полифенольных соединений экранировать свободные радикалы в клетках живых организмов. Экстраполяция данных, полученных в газовой фазе, на реакции в клеточной среде проводилась с помощью расчётов методами ТФП в рамках модели поляризуемого континуума. Предложен молекулярный механизм антиоксидантной активности полифенольных соединений вблизи дыхательной цепи митохондрий в условиях избыточного отрицательного заряда. А именно, предполагается, что молекулярный водород, действующий как селективный антиоксидант, может эффективно образовываться в результате захвата электронов («утекающих» с дыхательной цепи митохондрий в межмембранное пространство) молекулами полифенольного соединения и, таким образом, может обуславливать антиоксидантную активность полифенола. Соответствующий отрицательно заряженный фрагмент, т.е. хиноидная структура, несущая на себе избыточной электрон, может служить в качестве переносчика электронов и возвратить захваченный электрон обратно в дыхательный цикл. Количество гидроксильных групп и их относительное расположение на ароматических кольцах полифенольной молекулы являются определяющими для предложенного механизма, поскольку этими характеристиками определяется величина сечения диссоциативного захвата электрона.

Ключевые слова. полифенолы, антиоксидантная активность, перенос электрона, отрыв атома Н, диссоциативный захват электрона, молекулярный механизм.

\section{1. Введение}

Митохондрии клеток живых организмов, являясь основным поставщиком внутриклеточной энергии, обладают также способностью генерировать т.н. активные формы кислорода (АФК), содержащие неспаренный электрон: супероксид анион-радикал $\left(\mathrm{O}_{2}^{\cdot-}\right)$ и гидроксильный радикал $\left(\mathrm{OH}^{\bullet}\right)$ [1-3]. АФК вырабатываются вблизи электрон-транспортной цепи митохондрий вследствие «утечки» электронов с белкового комплекса (убихинол-цитохром с-оксидоредуктаза), встроенного во внутреннюю мембрану митохондрий и участвующего в клеточном дыхании $[1,4]$. АФК обладают способностью вступать в реакции с ключевыми компонентами клеток, инициируя цепную реакцию разрушения липидных мембран и вызывая мутации ДНК, но при этом, используются организмом, например, для разрушения патогенных объектов и формирования иммунного ответа [5]. При нормальном функционировании организма, «неиспользованные» АФК должны быть деактивированы внутренними (в основном ферментативными) системами, тогда как нарушение баланса генерации/ утилизации АФК приводит к возникновению окислительного стресса, что по-видимому является главной причиной старения и сопутствующих заболеваний [6].

Кроме собственных защитных систем организма, нейтрализация АФК, по сути являющихся свободными радикалами, может быть осуществлена с помощью поступающих извне антиоксидантов. Хорошо известно, что такой способностью обладают полифенольные, т.е. содержащие несколько гидроксильных групп, соедине- ния природного происхождения, как например, флавоноиды растений и спинохромы морских ежей $[7,8]$. Принято считать, что молекулярные механизмы антиоксидантного действия полифенолов связаны с наличием ОН-групп и электрон-донорными свойствами, а именно, предлагаются две реакции: (1) отрыв атома водорода от $\mathrm{OH}$-группы и его присоединение к свободному радикалу и (2) перенос электрона от полифенола на активный свободный радикал [9]. В первом случае эффективность антиоксидантного действия должна коррелировать с энергией разрыва ОН-связи, тогда как во втором - с потенциалом ионизации полифенольной молекулы. Вместе с тем, известно, что большую роль в противорадикальной активности играют определённые структурные элементы (взаимное расположение ОН-групп) [7, 10], а сами молекулы полифенолов обладают также выраженными электрон-акцепторными свойствами $[11,12]$, при этом, полная картина их комплексного действия на организм далека от понимания [13].

Полагая, что нейтрализация АФК наиболее эффективна в области их генерации, т.е. вблизи дыхательной цепи митохондрий в условиях избыточного отрицательного заряда, ассоциируемого с «утечкой» электронов [14], в данной работе предложен механизм антиоксидантной активности полифенолов, связанный с их электрон-акцепторными свойствами. На основе экспериментальных данных по резонансному диссоциативному захвату электронов (ДЗЭ) молекулами полифенолов, выявлена отличительная особенность этого процесса, характерная именно для молекул с несколькими гидроксильными группами, которая может быть ответственна 
за их антиоксидантную активность. Предлагаемый подход основан на следующей аналогии. Хорошо известно, что образование $\mathrm{O}_{2}{ }^{--}$в митохондриях происходит путём одноэлектронного восстановления молекулярного кислорода электронами, «утёкшими» с дыхательной цепи в межмембранное пространство [1-5, 14]. Такой процесс может трактоваться, как захват электрона молекулой $\mathrm{O}_{2}$, которая имеет положительное сродство к электрону, равное 0.45 эВ [15]. Внешняя мембрана митохондрий является проницаемой для молекул с массами менее 1500 а.е.м. [16], т.е. для всех полифенольных антиоксидантов растительного происхождения, а любая молекула со сродством к электрону не менее, чем у $\mathrm{O}_{2}$, может конкурировать [17] с кислородом за захват электронов дыхательной цепи.

Разумеется, реакции в межмембранном пространстве происходят в цитозоле при физиологических температурах с участием сольватированных электронов и не могут быть полностью идентичны процессам резонансного захвата электронов, происходящим в вакууме. Однако известно, что основные особенности ДЗЭ сохраняются при переходе от газовой фазы к конденсированному состоянию [18], в том числе, сечение ДЗЭ может возрастать на несколько порядков величины при внедрении молекулы-мишени в кластеры воды [19]. Процессы вблизи электрон-транспортной цепи митохондрий происходят на межфазных границах, т.е. именно там, где обнаружены электроны, сольватированные с небольшими энергиями связи (1.6 эВ в сравнении с 3.3. эВ для электронов, находящихся в объеме), которые попадают в «энергетический коридор», характерный для ДЗЭ [20]. Кроме того, о возможном проявлении ДЗЭ в клетках свидетельствуют факты совпадения продуктов метаболизма и фрагментов распада отрицательных ионов некоторых биологически активных веществ [21, 22].

В данной работе в качестве объектов исследования выбраны полифенольные стильбены растительного происхождения - ресвератрол и пицеатаннол (структуры приведены на рис.1), проявляющие выраженную антиоксидантную активность [23]. На основе экспериментальных данных, полученных в газовой фазе, высказано предположение о возможном поведении этих молекул в межмембранном пространстве митохондрий при взаимодействии с электронами дыхательной цепи. С привлечением ранее полученных данных для флавоноидов и спинохромов, предложен новый механизм антиоксидантной активности полифенольных соединений.<smiles></smiles>

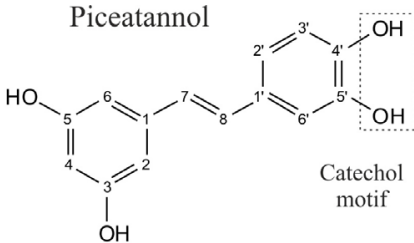

Рис. 1. Структура молекул ресвератрола $\left(\mathrm{C}_{14} \mathrm{H}_{12} \mathrm{O}_{3}\right)$ и пицеатаннола $\left(\mathrm{C}_{14} \mathrm{H}_{12} \mathrm{O}_{4}\right)$. Указана нумерация атомов и катехольная группа пицеатаннола.

Fig. 1. Molecular structure of resveratrol $\left(\mathrm{C}_{14} \mathrm{H}_{12} \mathrm{O}_{3}\right)$ and piceatannol $\left(\mathrm{C}_{14} \mathrm{H}_{12} \mathrm{O}_{4}\right)$. Atom labeling and catechol motif in piceatannol are shown.

\section{2. Экспериментальные и расчётные методы}

Используемая экспериментальная техника, а также вычислительные методы, подробно описаны в приложении к исследованию биологически активных молекул в работе [24]. Резонансный захват электронов молекулами (М), приводящий к образованию отрицательных молекулярных ионов $\left(\mathrm{M}^{-*}\right)$ и их последующему распаду с образованием ряда заряженных и нейтральных фрагментов, т.е. процесс $\mathrm{M}+\mathrm{e}^{-} \rightarrow \mathrm{M}^{-\star} \rightarrow$ Фрагменты, исследовался методом спектроскопии ДЗЭ [25]. Метод также известен в отечественной литературе, как массспектрометрия отрицательных ионов резонансного захвата электронов [26]. Пучок электронов заданной энергии в диапазоне 0-14 эВ (полуширина распределения по энергии около 0.4 эВ) пропускался через ячейку столкновений, заполненную газом исследуемого соединения в условиях парных столкновений. Токи образующихся отрицательных ионов, сепарированные по массе с помощью секторного магнитного поля, регистрировались в зависимости от энергии электронов в облучающем пучке. Калибровка шкалы энергии электронов осуществлялась по току анионов $\mathrm{SF}_{6}^{-}$, образованных захватом тепловых электронов молекулами гексафторида серы, а также $\mathrm{O}^{-}$из $\mathrm{CO}_{2}$ (резонансы при 4.3 и 8.1 эВ). Исследованные образцы ресвератрола и пицеатаннола были приобретены в компании Сигма-Алдрич, продукты R5010 и Р0453, соответственно. Вещества испарялись в ячейку столкновений при температуре $170^{\circ} \mathrm{C}$.

Интерпретация положений резонансных пиков проводилась с помощью расчётов методом теории функционала плотности с помощью пакета Gaussian 09 [27]. Энергии вакантных молекулярных орбиталей нейтральной молекулы определялись методом B3LYP/6-31G(d) и линейно масштабировались [28], для определения энергий вертикального захвата электрона [25]. Такой метод позволяет адекватно предсказывать положения резонансов формы $[25,26]$ в газовой фазе, а также максимумы плотности незаполненных электронных состояний при адсорбции на поверхностях [29-31]. Для оценок сродства молекул к электрону по разности полных энергий молекулы и аниона, а также оценки термодинамических порогов образования фрагментов при ДЗЭ, использовался базисный набор 6-31+G(d) с минимальным добавлением диффузных функций. Для расчёта энергий отрицательных ионов в водном растворе, с целью моделирования клеточных реакций, использовался метод поляризуемого континуума [32].

\section{3. Результаты}

Токи масс-сепарированных отрицательных ионов, образованных при взаимодействии электронов с молекулами ресвератрола и пицеатаннола, приведены в зависимости от энергии электронов на рис.2 (в порядке убывания интенсивности). Энергии максимумов, относительные интенсивности образования анионов, а также наиболее вероятные структуры отрицательно заряженных фрагментов указаны в табл.1 (в порядке убывания массового 
числа). В спектрах ДЗЭ обоих соединений наблюдаются долгоживущие (десятки микросекунд - время пролёта ионов через масс-спектрометр до регистрации) отрицательные молекулярные ионы (ОМИ) ресвератрола $(\mathrm{m} / \mathrm{e}=228)$ и пицеатаннола $(\mathrm{m} / \mathrm{e}=244)$, образованные при тепловой (0.0 эВ) энергии электронов. Токи ОМИ, наблюдаемые выше тепловых энергий обусловлены изотопными вкладами (изображены на рис.1 пунктирными линиями) от более интенсивных каналов распада ОМИ с выбросом атома водорода, т.е. образованием фрагментов $[\mathrm{M}-\mathrm{H}]^{-}$с массовыми числами $\mathrm{m} / \mathrm{e}=227$ и $\mathrm{m} / \mathrm{e}=243$ для ресвератрола и пицеатаннола, соответственно. Ток отрицательных ионов $[\mathrm{M}-\mathrm{H}]^{-}$, образующихся при энергии 1.2 эВ, является наиболее интенсивным в спектре ДЗЭ ресвератрола. Немного меньшую

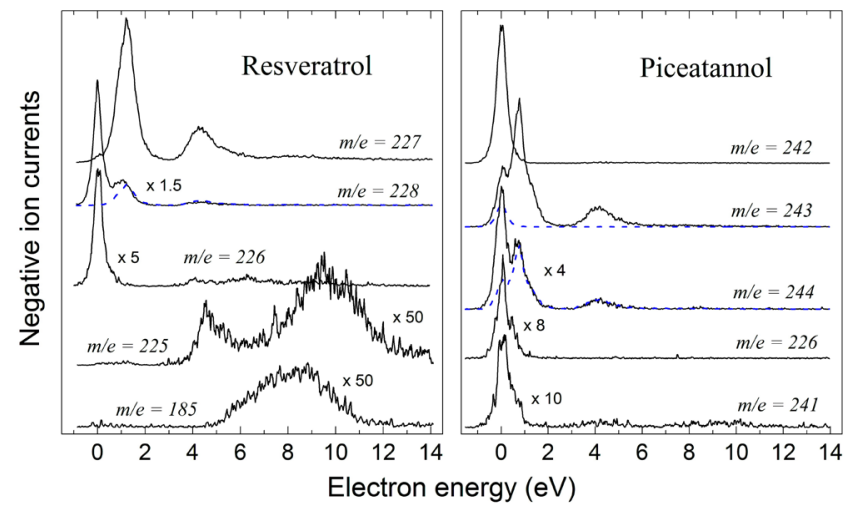

Рис. 2. Спектры ДЗЭ ресвератрола и пицеатаннола. Пунктирными линиями показаны изотопные вклады.

Fig. 2. Dissociative electron attachment spectra for resveratrol and piceatannol. Isotopic contributions are shown by dashed lines. интенсивность имеет канал выброса двух атомов водорода из ОМИ ресвератрола, т.е. фрагмент [M - 2H]$(\mathrm{m} / \mathrm{e}=226)$, наблюдаемый при тепловой энергии электронов. В пицеатанноле интенсивности этих каналов распада инвертированы, т.е. образование [M - 2H]$(\mathrm{m} / \mathrm{e}=242)$ при тепловой энергии заметно превышает интенсивность выброса одного атома Н из ОМИ $(\mathrm{m} / \mathrm{e}=243)$, наблюдаемого при 0.8 эВ. Со значительно меньшими интенсивностями наблюдаются другие распады: выброс трёх атомов водорода $[\mathrm{M}-3 \mathrm{H}]^{-}$, разрыв ароматического кольца $(\mathrm{m} / \mathrm{e}=185)$ в ресвератроле и отрыв гидроксильной группы и атома Н в пицеатанноле (m/e=226). В спектре ресвератрола эти распады наблюдаются только при высокой энергии электронов.

В табл.2 представлены данные расчётов методом B3LYP/6-31+G(d) термодинамических порогов появления для наиболее интенсивных распадов, ассоциируемых с отрывом одного и двух атомов водорода (для различных положений в молекуле). Согласно расчётам, образование анионов $[\mathrm{M}-\mathrm{H}]^{-}$возможно уже при энергии электронов 0.62 (ресвератрол) и 0.52 эВ (пицеатаннол), при условии, что отрыв Н происходит от гидроксильных групп в позициях 4' и 5', соответственно. Наиболее вероятно, что этими разрывами О-Н связей и обусловлены наблюдаемые токи $[\mathrm{M}-\mathrm{H}]-$ с максимумами при 1.2 (ресвератрол) и 0.8 эВ (пицеатаннол). Из-за большей прочности С-Н связей, отрывы атома Н от ароматического кольца (положение 4), либо от $\mathrm{C}=\mathrm{C}$ мостика (положение 7), требуют более 2.5 эВ, и могут соответствовать пикам, наблюдаемым около 4 эВ на токах анионов $[\mathrm{M}-\mathrm{H}]^{-}$.

Наибольший интерес для данной работы представляет достаточно редко наблюдаемое при ДЗЭ образова-

Таблица 1. Структуры анионов, наблюдаемых в масс-спектрах ресвератрола и пицеатаннола. Энергии максимумов (эВ) и относительные интенсивности.

Table 1. Structures of anions observed in the mass spectra of resveratrol and piceatannol. Peak energies (eV) and relative intensities. (sh. means shoulder)

\begin{tabular}{|c|c|c|c|}
\hline $\mathrm{M} / \mathrm{e}$ & $\begin{array}{l}\text { Структура аниона } \\
\text { Anion structure }\end{array}$ & $\begin{array}{c}\text { Энергия максимума } \\
\text { Peak energy }\end{array}$ & $\begin{array}{c}\text { Относительная интенсивность } \\
\text { Relative intensity }\end{array}$ \\
\hline \multicolumn{4}{|c|}{$\begin{array}{l}\text { Ресвератрол } \\
\text { Resveratrol }\end{array}$} \\
\hline 228 & $\mathrm{M}^{-}$ & 0.0 & 45 \\
\hline \multirow[t]{2}{*}{227} & {$[\mathrm{M}-\mathrm{H}]^{-}$} & 1.2 & 100 \\
\hline & & 4.3 & 24 \\
\hline \multirow[t]{2}{*}{226} & {$[\mathrm{M}-2 \mathrm{H}]^{-}$} & 0.0 & 17 \\
\hline & & 0.6 (плечо/sh.) & \\
\hline \multirow[t]{2}{*}{225} & {$[\mathrm{M}-3 \mathrm{H}]^{-}$} & 4.5 & 0.8 \\
\hline & & 9.5 & 1.3 \\
\hline 185 & {$\left[\mathrm{M}-(\mathrm{CH})_{2} \mathrm{OH}\right]^{-}$} & 8.7 & 0.8 \\
\hline \multicolumn{4}{|c|}{ Пицеатаннол } \\
\hline 244 & $\mathrm{M}^{-}$ & 0.0 & 16 \\
\hline 243 & {$[\mathrm{M}-\mathrm{H}]^{-}$} & 0.8 & 90 \\
\hline & & 4.0 & 13 \\
\hline 242 & {$[\mathrm{M}-2 \mathrm{H}]^{-}$} & 0.0 & 100 \\
\hline 241 & {$[\mathrm{M}-3 \mathrm{H}]^{-}$} & 0.0 & 6.5 \\
\hline 226 & {$[\mathrm{M}-\mathrm{H}-\mathrm{OH}]^{-}$} & $\begin{array}{l}0.6 \text { (плечо/sh.) } \\
0.0 \\
0.6 \text { (плечо/sh.) }\end{array}$ & 7.5 \\
\hline
\end{tabular}


ние фрагментов $[\mathrm{M}-2 \mathrm{H}]-$. Насколько известно авторам, выброс двух атомов водорода из ОМИ наблюдается (при низких энергиях электронов) лишь в тех случаях, когда молекула-мишень имеет определённую структуру: либо содержит несколько гидроксильных групп (полифенол) $[11,12,33]$, либо существует возможность внутренних вращений одних частей молекулы по отношению к другим, при которых происходит сближение атомов водорода [34]. В обоих случаях необходимо, чтобы в качестве нейтрального фрагмента образовалась молекула водорода: только при таком условии удаётся объяснить факт наблюдения анионов $[\mathrm{M}-2 \mathrm{H}]^{-}$при низких (вплоть до тепловых) энергиях электронов. Действительно, согласно расчёту (см. табл.2), при отрыве двух атомов Н от гидроксильных групп в положениях $(4,5)$ в ресвератроле и $\left(4^{\prime}, 5^{\prime}\right)$ в пицеатанноле, процесс образования [M - 2H]является наиболее предпочтительным с энергетической точки зрения и происходит с выигрышем энергии 0.93 и 1.08 эВ, соответственно. При этом фрагментарные отрицательные ионы имеют хиноидную структуру, как показано на рис.3. Отметим, что отрыв двух атомов водо- рода в положениях $(3,5)$ в ОМИ ресвератрола является более предпочтительным с кинетической точки зрения, поскольку при этом не требуется миграции атомов $\mathrm{H}$ навстречу друг другу для образования нейтральной молекулы водорода, но и освобождаемая энергия при этом меньше (0.55 эВ). Катехольная группа пицеатаннола (см. рис.1), в отличие от ресвератрола, легко позволяет обра-
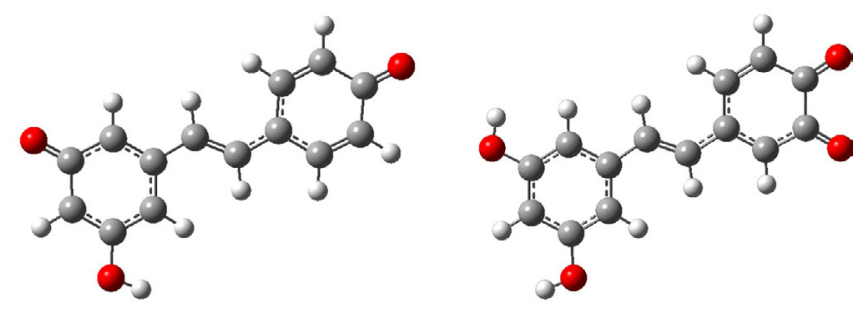

Рис. 3. Структура наиболее стабильных фрагментарных анионов $[\mathrm{M}-2 \mathrm{H}]^{-}$ресвератрола и пицеатаннола по данным расчёта методом B3LYP/6-31+G(d) в вакууме.

Fig. 3. The most stable B3LYP/6-31+G(d) structures of fragment anions $[\mathrm{M}-2 \mathrm{H}]^{-}$for resveratrol and piceatannol in vacuum.

Таблица 2. Полные энергии (эВ) относительно основного состояния нейтральных молекул ресвератрола и пицеатаннола, рассчитанные методом B3LYP/6-31+G(d) с учётом нулевых колебаний в изолированном состоянии (вакууме) и в водном растворе. Нумерация атомов соответствует рис. 1 .

Table 2. B3LYP/6-31+G(d) total energies $(\mathrm{eV})$ relative to that of the neutrall ground state of resveratrol and piceatannol corrected for zeropoint energy in the gas phase and water solvent. Atom labeling is reported in Fig.1.

\begin{tabular}{|c|c|c|c|}
\hline \multirow[t]{2}{*}{$\mathrm{M} / \mathrm{e}$} & \multirow{2}{*}{$\begin{array}{l}\text { Фрагменты } \\
\text { Fragments }\end{array}$} & \multicolumn{2}{|c|}{$\begin{array}{c}\text { Относительная энергия } \\
\text { Relative energy }\end{array}$} \\
\hline & & \multirow[t]{2}{*}{$\begin{array}{c}\text { вакуум } \\
\text { gas-phase }\end{array}$} & \multirow[t]{2}{*}{$\begin{array}{l}\text { вода } \\
\text { water }\end{array}$} \\
\hline \multicolumn{2}{|c|}{$\begin{array}{l}\text { Ресвератрол } \\
\text { Resveratrol }\end{array}$} & & \\
\hline 228 & $\mathrm{M}_{-}^{-}$ & $=0.54$ & $=2.20$ \\
\hline 227 & {$\left[\mathrm{M}-\mathrm{H}\left(4^{\prime}\right)\right]^{-}+\mathrm{H}^{\bullet}$} & 0.62 & -1.21 \\
\hline 227 & {$[\mathrm{M}-\mathrm{H}(5)]^{-}+\mathrm{H}^{\bullet}$} & 0.98 & -1.10 \\
\hline 227 & {$[\mathrm{M}-\mathrm{H}(3)]^{-}+\mathrm{H}^{\bullet}$} & 0.96 & -1.10 \\
\hline 227 & {$[\mathrm{M}-\mathrm{H}(4)]^{-}+\mathrm{H}^{\bullet}$} & 2.67 & 0.63 \\
\hline 227. & {$[\mathrm{M}=\mathrm{H}(7)]_{--}+\mathrm{H}^{\circ}$} & - 2.74 & _ 0.88 \\
\hline 226 & {$\left[\mathrm{M}-2 \mathrm{H}\left(4^{\prime}, 5\right)\right]^{-}+\mathrm{H}_{2}$} & -0.93 & -2.61 \\
\hline 226 & {$\left[\mathrm{M}-2 \mathrm{H}\left(3,4^{\prime}\right)\right]^{-}+\mathrm{H}_{2}^{2}$} & -0.93 & -2.62 \\
\hline 226 & {$[\mathrm{M}-2 \mathrm{H}(3,5)]^{-}+\mathrm{H}_{2}$} & -0.55 & -2.57 \\
\hline 226 & {$\left[\mathrm{M}-2 \mathrm{H}\left(2,2^{\prime}\right)\right]^{-}+\mathrm{H}_{2}$} & -0.37 & -1.99 \\
\hline 226 & {$[\mathrm{M}-2 \mathrm{H}(7,8)]^{-}+\mathrm{H}_{2}$} & 1.19 & -0.49 \\
\hline \multicolumn{4}{|c|}{ Пицеатаннол } \\
\hline \multicolumn{4}{|c|}{ Piceatannol } \\
\hline 244 & $\mathrm{M}_{-}^{-} \ldots \ldots \ldots$ & -0.56 & -2.22 \\
\hline 243 & {$\left[\mathrm{M}-\mathrm{H}\left(5^{\prime}\right)\right]^{-}+\mathrm{H}^{\cdot}$} & 0.52 & -1.32 \\
\hline 243 & {$\left[\mathrm{M}-\mathrm{H}\left(4^{\prime}\right)\right]^{-}+\mathrm{H}^{\cdot}$} & 0.83 & -1.14 \\
\hline 243 & {$[\mathrm{M}-\mathrm{H}(5)]^{-}+\mathrm{H}^{\bullet}$} & 0.91 & -1.11 \\
\hline 243 & {$[\mathrm{M}-\mathrm{H}(3)]^{-}+\mathrm{H}^{\bullet}$} & 0.97 & -1.09 \\
\hline 243 & {$[\mathrm{M}-\mathrm{H}(4)]^{-}+\mathrm{H}^{\bullet}$} & 2.64 & 0.62 \\
\hline 243 & {$[\mathrm{M}-\mathrm{H}(7)]_{-}^{-}+\mathrm{H}^{\cdot}$} & 3.03 & 0.88 \\
\hline 242 & {$\left[\mathrm{M}-2 \mathrm{H}\left(4^{\prime}, 5^{\prime}\right)\right]^{-}+\mathrm{H}_{2}$} & -1.08 & -3.06 \\
\hline 242 & {$\left[\mathrm{M}-2 \mathrm{H}\left(5,5^{\prime}\right)\right]^{-}+\mathrm{H}_{2}$} & -1.04 & -2.64 \\
\hline 242 & {$\left[\mathrm{M}-2 \mathrm{H}\left(3,5^{\prime}\right)\right]^{-}+\mathrm{H}_{2}^{2}$} & -0.93 & -2.62 \\
\hline 242 & {$\left[\mathrm{M}-2 \mathrm{H}\left(4^{\prime}, 5\right)\right]^{-}+\mathrm{H}_{2}$} & -0.82 & -2.66 \\
\hline 242 & {$\left[\mathrm{M}-2 \mathrm{H}\left(3,4^{\prime}\right)\right]^{-}+\mathrm{H}_{2}$} & -0.77 & -2.64 \\
\hline 242 & {$[\mathrm{M}-2 \mathrm{H}(3,5)]^{-}+\mathrm{H}_{2}$} & -0.58 & -2.57 \\
\hline 242 & {$[\mathrm{M}-2 \mathrm{H}(7,8)]^{-}+\mathrm{H}_{2}$} & 1.13 & -0.51 \\
\hline
\end{tabular}


зовать $\mathrm{H}_{2}$ и $[\mathrm{M}-2 \mathrm{H}]-$, чем, по-видимому, и обусловлена бо́льшая относительная интенсивность этого распада, даже по сравнению образованием иона $[\mathrm{M}-\mathrm{H}]^{-}$путём простого разрыва одной связи.

Наиболее интенсивные процессы образования и распада ОМИ исследованных молекул наблюдаются при энергиях менее 2 эВ (см. рис.2). Как правило, резонансные состояния отрицательных ионов, определяющие положения максимумов спектра ДЗЭ при энергиях меньше 3-4 эВ, образуются по механизму резонанса формы [25, 26] захватом электрона на одну из вакантных молекулярных орбиталей (MO) л-типа, поскольку такие состояния являются более долгоживущими, нежели $\sigma$-состояния. Согласно расчётам, молекулы исследованных соединений имеют по четыре вакантных МО $\pi$-типа в диапазоне энергий до 2 эВ. Формы, практически совпадающие для ресвератрола и пицеатаннола, а также расчётные энергии этих $\mathrm{MO}$ представлены на рис.4. Относительно широкие пики токов $[\mathrm{M}-\mathrm{H}]-$ около 1 эВ, по видимому, содержат вклады (неразрешённые в данном эксперименте) от орбиталей $\pi_{2}^{*}-\pi_{4}^{*}$. Из них, только орбиталь $\pi_{2}^{*}$ заметно дестабилизована в пицеатанноле по сравнению с ресвератролом, вероятно по причине её локализации на ароматическом кольце с дополнительной ОН-группой. Положения остальных МО в исследованных молекулах практически совпадают. Нижняя вакантная $\mathrm{MO}\left(\pi_{1}^{*}\right)$, делокализованная по всей молекуле, включая вклад $\mathrm{C}=\mathrm{C}$ мостика, расположена в связанной области и ответственна за захват тепловых электронов и наблюдаемые токи анионов при 0.0 эВ. Наблюдение долгоживущих ОМИ исследуемых молекул при тепловой энергии находится в согласии с предсказанными значениями сродства к электрону, равными 0.54 и 0.56 эВ (см. табл.2) для ресвератрола и пицеатаннола, соответственно.

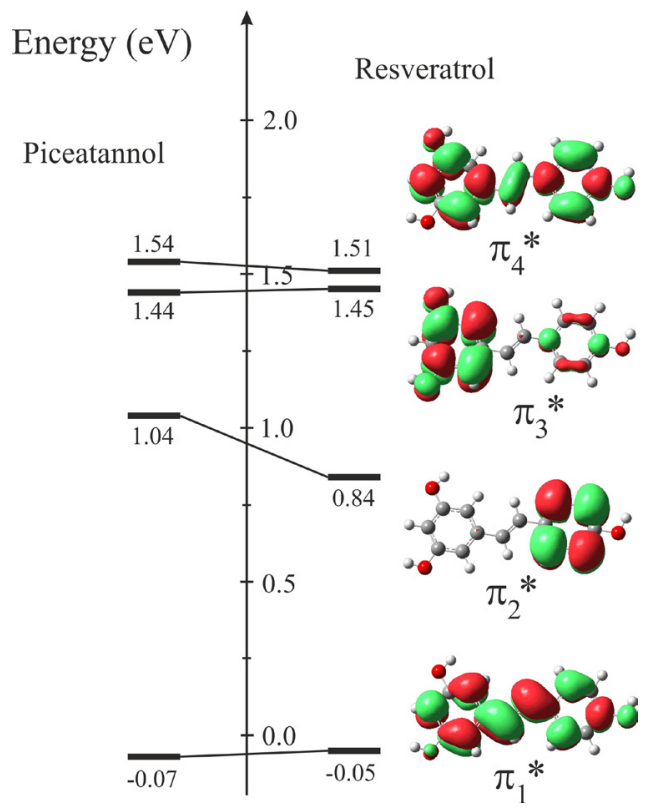

Рис. 4. Схематическое изображение и энергии первых четырёх вакантных МО л-типа в ресвератроле и пицеатанноле по данным расчёта методом B3LYP/6-31G(d) и масштабирования.

Fig. 4. Schematic representation and the scaled energies for the lowest four vacant $\pi$ molecular orbitals in resveratrol and piceatannol calculated on B3LYP/6-31G(d) level.

\section{4. Обсуждение}

Руководствуясь полученными в газовой фазе экспериментальными данными, а также фактами, свидетельствующими о подобии процессов ДЗЭ в вакууме и конденсированном состоянии [18-20], рассмотрим гипотетические особенности захвата электронов молекулами ресвератрола и пицеатаннола в сольватированном состоянии. Последний процесс будем считать моделью биохимических реакций вблизи дыхательной цепи митохондрий в условиях избыточного отрицательного заряда. Поскольку в растворе происходит перенос (туннелирование) связанных электронов, то наиболее вероятен их захват на нижнюю вакантную МО, хотя состояния с энергиями до 1-1.5 эВ (в газовой фазе), также могут быть доступны [35]. Захват свободных электронов на орбиталь $\pi_{1}^{*}$ исследуемых молекул в вакууме приводит к конкурирующим процессам образования ОМИ и фрагментации на $[\mathrm{M}-2 \mathrm{H}]^{-}$и $\mathrm{H}_{2}$, причём в пицеатанноле распад имеет бо́льшую относительную интенсивность, благодаря наличию катехольной группы. Поскольку «движущей силой» реакций в растворах является разность энергий реагентов и продуктов [36], отметим, что согласно расчётам (см. табл.2) образование $[\mathrm{M}-2 \mathrm{H}]-$ и $\mathrm{H}_{2}$ является самым выгодным распадом в водной среде с энергетической точки зрения, причём конечные продукты на 0.4 и 0.8 эВ более стабильны, чем ОМИ, соответственно, ресвератрола и пицеатаннола. Интересно, что пространственная удалённость $\mathrm{OH}$-групп друг от друга в ресвератроле нивелируется для распада в растворе: отрыв атомов водорода от любой пары гидроксилов приводит, в отличие от газовой фазы, примерно к одинаковому выигрышу энергии 2.6 эВ (см. табл.2).

Приведенные соображения позволяют утверждать, что перенос сольватированных электронов на молекулы ресвератрола и пицеатаннола должен в основном приводить к характерной фрагментации с образованием $[\mathrm{M}-2 \mathrm{H}]^{-}$(хиноидная структура) и $\mathrm{H}_{2}$. Отметим, что аналогичный вывод может быть сделан для других полифенольных соединений - флавоноидов и спинохромов $[11,12]$, что независимо подтверждается результатами исследований методом электрораспыления [37]. На этой основе предлагается новый механизм биологической активности (благотворного действия на организм в условиях окислительного стресса) полифенольных соединений, связанный с характерной особенностью образования и распада их ОМИ. А именно, оказавшись в области генерации АФК вблизи электрон-транспортной цепи, молекулы полифенольного соединения могут вызвать следующие три положительных эффекта (рис.5):

1) часть «утекающих» с дыхательной цепи электронов захватывается молекулами полифенола, что исключает их взаимодействие с клеточным кислородом и образование супероксид-радикала, дающего начало генерации других АФК;

2) молекулы $\mathrm{H}_{2}$ (терапевтический газ), образующиеся в межмембранном пространстве, являются сильнейшим универсальным антиоксидантом селективного действия $[38,39]$, который не вмешивается в работу им- 


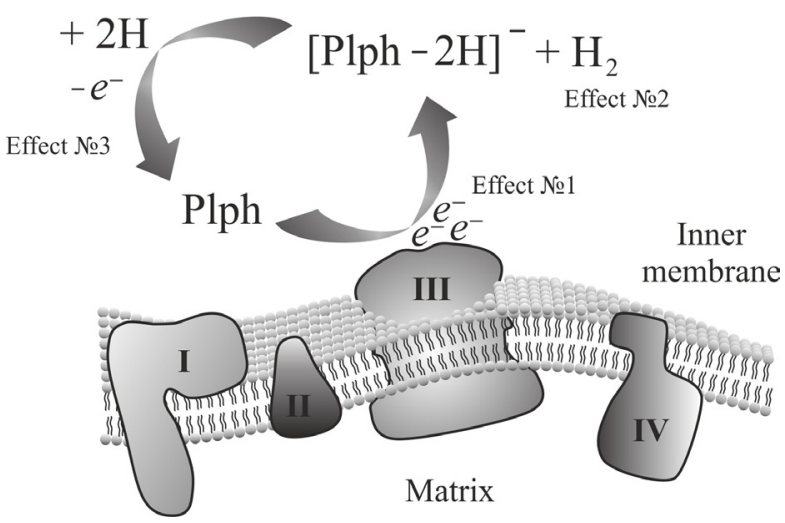

Рис. 5. Предполагаемый механизм трансформации молекулы полифенольного соединения (Plph) в межмембранном пространстве митохондрии после взаимодействия с электроном дыхательной цепи. I-IV - ферментативные комплексы цепи переноса электронов.

Fig. 5. Suggested mechanism for transformation of polyphenolic molecule (Plph) in mitochondrial intermembrane space following its interaction with an electron derived from the electron-transfer chain. I-IV - enzymatic complexes of the respiratory chain.

мунной системы и имеет широчайший спектр полезного действия на организм [40];

3) хиноидная структура, несущая на себе избыточный электрон, может «вернуть» его обратно в дыхательную цепь, подобно другим восстановителям клеточного дыхания хиноидной природы [41], а также, в соответствии с ролью хинонов, как переносчиков электронов в клетках растений [42]; при этом возможно циклическое действие полифенольного соединения.

T.о. в рамках предложенного механизма антиоксидантное действие полифенольных соединений в основном сводится к выделению $\mathrm{H}_{2}$ в области генерации АФК (эффект №2 на рис.5), и определяется электрон-акцепторными свойствами:

1) сродство к электрону должно быть достаточным для конкуренции с $\mathrm{O}_{2}$ за электроны, но не являться слишком большим, как в случае некоторых хинонов или галогензамещённых молекул, что чревато сильным вмешательством в работу дыхательной цепи (токсический эффект), из-за прочного связывания электрона в ОМИ без его диссоциации [35], либо эффективного образования токсичных радикалов и деактивации ферментов [43];

2) сечение ДЗЭ с образованием хинона $[\mathrm{M}-2 \mathrm{H}]^{-}$ и $\mathrm{H}_{2}$ должно быть велико, что в свою очередь, отчасти определяется количеством и взаимным расположением гидроксильных групп.

Например, катехольная группа играет ключевую роль в противорадикальной активности [7], и, в нашем случае, обеспечивает более эффективное образование $[\mathrm{M}-2 \mathrm{H}]^{-}$и $\mathrm{H}_{2}$ в пицеатанноле. Последний, действительно, является более эффективным антиоксидантом, чем ресвератрол [44-47], несмотря на то, что наблюдаемые эффекты полифенольных соединений в организме очень многогранны, и определяются множеством факторов, как например, биодоступностью и эффективностью усвоения.

\section{5. Заключение}

На основе исследований резонансного диссоциативного захвата электронов молекулами полифенольных стильбенов растительного происхождения (ресвератрол и пицеатаннол) показано, что их биологическая активность вблизи дыхательной цепи митохондрий может в значительной степени определяться электрон-акцепторными свойствами. Принимая во внимание аналогичные данные, полученные для других классов полифенольных соединений (флавоноиды и спинохромы), предложен новый механизм их антиоксидантной активности, основанный на характерной диссоциации отрицательных ионов полифенолов с образованием молекулярного водорода и хиноидной структуры, несущей избыточный электрон. Выдвинутые предположения представляют собой новый подход к рассмотрению биохимических процессах в клетках, происходящих в условиях избыточного отрицательного заряда вблизи электрон-транспортных путей в митохондриях млекопитающих и тилакоидах растений.

Благодарность. Работа выполнена при финансовой поддержке Санкт-Петербургского государственного университета (грант СПбГУ ИАС: 11.38.219.2014) и Российского фонда фундаментальных исследований (гранты № 15-29-05786-oø u_M, 15-02-02809-a, 14-03-00087-a).

Acknowledgements. Thanks are due to Saint-Petersburg State University (research grant 11.38.219.2014) and the Russian Foundation for Basic Research (grants \#15-29-05786, \#14-03-00087 and \#15-02-02809) for financial support.

\section{Литература}

1. И.Н. Тодоров, Митохондрии: окислительный стресс и мутации митохондриальной ДНК в развитии патологий, процессе старения и апоптозе, Российский химический журнал, 51(1), 93-106 (2007).

2. Е.Б. Меньщикова, В.З. Ланкин, Н.К. Зенков, И.А. Бондарь, Н.Ф. Круговых, В.А. Труфакин, Окислительный стресс. Прооксиданты и антиоксиданты, М.: Фирма «Слово», 2006, 556 с.

3. В.Г. Гривенникова, А.Д. Виноградов, Генерация активных форм кислорода митохондриями. Успехи биол. химии, 53, 245-296 (2013).

4. Q. Chen, E.J. Vazquez, S. Moghaddas, C.L. Hoppel, E.J. Lesnefsky, Production of reactive oxygen species by mitochondria. Central role of Complex III, Journal of Biological Chemistry, 278(38), 36027-36031 (2003).

5. В.И. Донцов, В.Н. Крутько, Б.М. Мрикаев, С.В. Уханов, Активные формы кислорода как система: значение в физиологии, патологии и естественном старении, Труды ИСА РАН, 19, 50-69 (2006).

6. В.П. Скулачев, Попытка биохимиков атаковать проблему старения: “Мегапроект" по проникающим ионам. Первые итоги и перспективы (обзор), Биохимия, 72(12), 1700-1714 (2007). 
7. Ю.С. Тараховский, Ю.А. Ким, Б.С. Абдрасилов, Е.Н. Музафаров, Флавоноиды: Биохимия, биофизика, медицина, Пущино: Synchrobook (2013), 310 стр.

8. R. Kuwahara, H. Hatate, T. Yuki, H. Murata, R. Tanaka, Y. Hama, Antioxidant property of polyhydroxylated naphthoquinone pigments from shells of purple sea urchin Anthocidaris crassispina, LWT-Food Science and Technology, 42(7), 1296-1300 (2009).

9. M. Leopoldini, N. Russo, M. Toscano, The molecular basis of working mechanism of natural polyphenolic antioxidants, Food Chemistry, 125(2), 288-306 (2011).

10. А.М. Попов, О.Н. Кривошапко, А.А. Артюков, Механизмы протективной фармакологической активности флавоноидов, Биофармацевтический журнал, 4(4), 27-41 (2012).

11. A. Modelli, S.A. Pshenichnyuk, Gas-phase dissociative electron attachment to flavonoids and possible similarities to their metabolic pathways, Phys. Chem. Chem. Phys. $15,1588-1600$ (2013).

12. N.L. Asfandiarov, S.A. Pshenichnyuk, A.S. Vorob'ev, E.P. Nafikova, Y.N. Elkin, D.N. Pelageev, E.A. Koltsova, A. Modelli, Electron attachment to some naphthoquinone derivatives: Long-lived molecular anion formation, Rapid Communications in Mass Spectrometry, 28, 1580-1590 (2014).

13. A.A. Kamboh, M.A. Arain, M.J. Mughal, A. Zaman, Z.M. Arain, A.H. Soomro, Flavonoids: health promoting phytochemicals for animal production, J. Anim. Health Prod, 3(1), 6-13 (2015).

14. Г.Г. Мартинович, С.Н. Черенкевич Окислительновосстановительные процессы в клетках: Минск: БГУ, 2008. c. 159.

15. K.M. Ervin, I. Anusiewicz, P. Skurski, J. Simons, W.C. Lineberger, The only stable state of $\mathrm{O} 2$ - is the $\mathrm{X} 2 \mathrm{Mg}$ ground state and it (still!) has an adiabatic electron detachment energy of $0.45 \mathrm{eV}$, The Journal of Physical Chemistry A 107, 8521-8529 (2003).

16. A. Szewczyk, L. Wojtczak, Mitochondria as a pharmacological target, Pharmacological reviews, 54(1), 101-127 (2002).

17. J.E. Biaglow, Cellular electron transfer and radical mechanisms for drug metabolism, Radiation Research 86, 212-242 (1981).

18. O. Ingólfsson, F. Weik, E. Illenberger, The reactivity of slow electrons with molecules at different degrees of aggregation: gas phase, clusters and condensed phase, Int. J. Mass Spec. Ion Proc. 155, 1-68 (1996).

19. I.I. Fabrikant, S. Caprasecca, G.A. Gallup, J.D. Gorfinkiel, Electron Attachment to Molecules in a Cluster Environment, J. Chem. Phys. 136, 184301-8 (2012).

20. K.R. Siefermann, Y. Liu, E. Lugovoy, O. Link, M. Faubel, U. Buck, B. Winter, B. Abel, Binding Energies, Lifetimes and Implications of Bulk and Interface Solvated Electrons in Water, Nature Chemistry 2, 274-279 (2010).

21. S.A. Pshenichnyuk, A. Modelli, Electron attachment to antipyretics: Possible implications of their metabolic pathways, Journal of Chemical Physics, 136(23), 234307 (2012).

22. S.A. Pshenichnyuk, A.S. Komolov, Dissociative electron attachment to anthralin to model its biochemical reactions, Journal of Physical Chemistry Letters, 5(16), 2916-2921 (2014).

23. H. Piotrowska, M. Kucinska, M. Murias, Biological activity of piceatannol: leaving the shadow of resveratrol, Mutation Research/Reviews in Mutation Research, 750(1), 60-82 (2012).

24. S.A. Pshenichnyuk, A. Modelli, ETS and DEAS Studies of the Reduction of Xenobiotics in Mitochondrial Intermembrane Space. Mitochondrial Medicine: Volume II, Manipulating Mitochondrial Function, 285-305 (2015).

25. E. Illenberger, J. Momigny, Gaseous Molecular Ions. An Introduction to Elementary Processes Induced by Ionization, Steinkopff Verlag Darmstadt, Springer-Verlag, New York, (1992).

26. В.И. Хвостенко, Масс-спектрометрия отрицательных ионов в органической химии, Москва, Наука, 1981.

27. M.J. Frisch, G.W. Trucks, H.B. Schlegel, G.E. Scuseria, M.A. Robb, J.R. Cheeseman, G. Scalmani, V. Barone, B. Mennucci, G.A. Petersson, et al., Gaussian 09, Revision A.02, Gaussian, Inc., Wallingford CT, 2009.

28. A.M. Scheer, P.D. Burrow, $\pi^{\star}$ Orbital System of Alternating Phenyl and Ethynyl Groups: Measurements and Calculations, J. Phys. Chem. B., 110, 17751-17756 (2006).

29. S.A. Pshenichnyuk, A.S. Komolov, Relation between electron scattering resonances of isolated NTCDA molecules and maxima in the density of unoccupied states of condensed NTCDA layers. J. Phys. Chem. A., 116, 761-766 (2011).

30. S.A. Pshenichnyuk, A.V. Kukhto, I.N. Kukhto, A.S. Komolov, Spectroscopic states of PTCDA negative ions and their relation to the maxima of unoccupied state density in the conduction band. Tech. Phys., 56, 754-759 (2011).

31. A.S. Komolov, E.F. Lazneva, S.A. Pshenichnyuk, N.S. Chepilko, A.A. Tomilov, N.B. Gerasimova, A.A. Lezov, P.S. Repin, Electronic properties of the interface between hexadecafluoro copper phthalocyanine and unsubstituted copper phthalocyanine films, Semiconductors, 47 (7), 956-961 (2013).

32. J. Tomasi, M. Persico, Molecular Interactions in Solution: An Overview of Methods Based on Continuous Distributions of the Solvent. Chemical Reviews, 94, 2027 2094 (1994).

33. S.A. Pshenichnyuk, Y.N. Elkin, N.I. Kulesh, E.F. Lazneva, A.S. Komolov, Low-energy electron interaction with retusin extracted from Maackia amurensis: towards a molecular mechanism of the biological activity of flavonoids, Phys. Chem. Chem. Phys. 17(26), 1680516812 (2015).

34. S.A. Pshenichnyuk, N.L. Asfandiarov, A.V. Kukhta, Interruption of the inner rotation initiated in isolated electron-driven molecular rotors, Physical Review A, 86(5), 052710 (2012).

35. L.G. Christophorou, D. Hadjiantoniou, Electron attachment and molecular toxicity, Chemical physics letters, 419(4), 405-410 (2006).

36. S. Antonello, F. Maran, Intramolecular dissociative electron transfer, Chemical Society Reviews, 34(5), 418428 (2005). 
37. L. Feketeová, C.K. Barlow, T.M. Benton, S.J. Rochfort, A.J. Richard, The formation and fragmentation of flavonoid radical anions, International Journal of Mass Spectrometry, 301(1), 174-183 (2011).

38. I. Ohsawa, M. Ishikawa, K. Takahashi, M. Watanabe, K. Nishimaki, K. Yamagata, K. Ken-ichiro Katsura, Y . Katayama, S. Asoh, S. Ohta, Hydrogen acts as a therapeutic antioxidant by selectively reducing cytotoxic oxygen radicals, Nature Medicine 13, 688-694 (2007).

39. C.S. Huang, T. Kawamura, Y. Toyoda, A. Nakao, Recent advances in hydrogen research as a therapeutic medical gas, Free radical research 44, 971-982 (2010).

40. Y. Hong, S. Chen, J.M. Zhang, Hydrogen as a selective antioxidant: A review of clinical and experimental studies, Journal of International Medical Research 38, 1893-1903 (2010).

41. Л.В. Селина, К.А. Мотовилов, Л.С. Ягужинский, К.И. Агладзе, Восстановление сократительной активности первичной культуры кардиомиоцитов крысы, подавленной ингибиторами митохондриальной NADH-дегидрогеназы, под влиянием гидрофильных хинонов. Труды МФТИ 5(1), 129-139 (2013).
42. W. Palladin, Die Atmungspigmente der Pflanzen, HoppeSeyler' s Zeitschrift für physiologische Chemie 55, 207 222 (1908).

43. N.L. Gregory, Carbon tetrachloride toxicity and electron capture, Nature 212, 1460-1461 (1966).

44. Z. Ovesná, K. Kozics, Y. Bader, P. Saiko, N. Handler, T. Erker, T. Szekeres, Antioxidant activity of resveratrol, piceatannol and 3, 3, 4, 4', 5, 5'-hexahydroxy-transstilbene in three leukemia cell lines, Oncology reports 16(3), 617-624 (2006).

45. M. Murias, W. Jäger, N. Handler, T. Erker, Z. Horvath, T. Szekeres, et al. Antioxidant, prooxidant and cytotoxic activity of hydroxylated resveratrol analogues: structureactivity relationship, Biochemical pharmacology 69(6), 903-912 (2005).

46. Z. Ovesna, K. Horvathova-Kozics, Structure-activity relationship of trans-resveratrol and its analogues, Neoplasma, 52(6), 450 (2005).

47. Y. Rhayem, P. Thérond, L. Camont, M. Couturier, J.L. Beaudeux, A. Legrand et al. Chain-breaking activity of resveratrol and piceatannol in a linoleate micellar model, Chemistry and physics of lipids, 155(1), 48-56 (2008). 